1 Validation of a phenomenological strain-gradient plasticity theory

2 D.J. Dunstan

3 School of Physics and Astronomy, Queen Mary University of London,

4 Address: London, E1 4NS, England

5 E-mail: d.dunstan@qmul.ac.uk

6 


\section{Validation of a phenomenological strain-gradient plasticity theory}

Strain-gradient plasticity theories have been developed to account for the size

effect in small-scale plasticity in metals. However, they remain of limited use in engineering, for example in standards for nanoindentation, because of their phenomenological nature. In particular, a key parameter, the characteristic length, can only be determined by fitting to experiment. Here it is shown that the characteristic length in one such theory derives directly from known quantities through fundamental dislocation physics. This explains and validates the theory for use in engineering.

Keywords: plasticity of metals; strengthening mechanisms; strained layers; dislocations; strain-gradient theory; critical thickness theory.

20 The increase in strength (the size effect) when dislocation-mediated plasticity is restricted

21 to small volumes has been extensively documented experimentally over the past 60 years

22 [see, e.g., 1-13]. It is an important effect in many technologies from metallurgy to

23 semiconductors, yet it is not fully understood $[12,14]$. In micromechanics, many loading

24 conditions impose a plastic strain gradient, and so theories in which the strain gradient

25 plays a central role have been developed [3-6, 15-19]. In contrast, in semiconductor

26 technology, Matthews critical thickness theory has been largely accepted to explain and

27 predict the effect in terms simply of the size - stronger when smaller [20-22]. The

28 strain-gradient theories have not been comprehensively embraced [23], because of

29 ambiguities about the underlying physics and about the parameters - in particular, the

30 characteristic length - which enter into these theories. One consequence is that there are

31 no satisfactory international standards for comparing nanoindentation data, in which the 
32 size effect plays an important role, with macroscopic indentation data. Here it is shown

33 that the Fleck-Hutchinson strain-gradient theory [4, 17-19, 23] follows mathematically

34 and physically directly from critical thickness theory [20-22]. The strain-gradient theory

35 fits experiment well, but with the characteristic length as a free fitting parameter. This

36 phenomenological parameter is here derived from known physical quantities via critical

37 thickness theory. The derivation and the associated re-interpretation validate the strain-

38 gradient theory for use in practical engineering contexts, as an approximation that

39 expresses a non-local property as a local property.

40 Increases in strength (the size effect) due to boundaries imposed on dislocation-

41 mediated plasticity on scales up to tens of microns have been presented for

42 nanoindentation [3,5], thin wires in torsion $[4,9,10]$, thin foils in bending [6, 8], and for a

43 large variety of still smaller structures down to sub-micron sizes mostly created by

44 focused ion-beam (FIB) milling [e.g. 7, 11, 13]. Microstructural constraints giving rise to

45 the size effect include sub-grain boundaries [2] and grain boundaries (the Hall-Petch

46 effect) [1, 12]. Pseudomorphic (strained-layer) heteroepitaxial crystal growth is another

47 key example [20-22]. In many of these situations, plastic strain gradients are necessarily

48 or optionally present, and there is widespread agreement that in such situations the size

49 effect can be attributed to the strain gradient.

50 In formal continuum mechanics, to set up a strain-gradient plasticity theory

51 (SGP), the stress is not only a function of plastic strain $\varepsilon_{P}$, but also a function of its spatial

52 gradient $\ell \varepsilon_{P}^{\prime}=\ell \mathrm{d} \varepsilon_{P} / \mathrm{d} s$ where $s$ is position and the characteristic length $\ell$ is introduced

53 to give a dimensionless quantity [16-19]. Where a physical interpretation is called for, 
54 appeal is made to the geometrically-necessary dislocations (GNDs) [3] which in a

55 crystalline material are necessarily associated with plastic strain gradients [15]. Values of

$56 \ell$ are found from fitting to experiment (see Fig.1). The major problem for such strain-

57 gradient theories is to give a reasonable physical interpretation of the values of $\ell$ that

58 result. There have been many proposals. See [24] for a recent discussion and a new

59 proposal.

Evans and Hutchinson [23] gave an appraisal of SGP theories, for brevity

61 confined to the Nix-Gao (NG) theory [3] and the Fleck-Hutchinson (FH) theory [4, 17,

62 19]. These two theories illustrate adequately both the successes of SGP theories in

63 general, and their difficulties. The successes lie in the good fits to experimental data that

64 these theories give. The major difficulty is that, fitting to experimental datasets for soft

65 metals, the NG theory gives characteristic lengths $\ell_{\mathrm{NG}} \sim 25 \mathrm{~mm}$, and the FH theory gives

$66 \ell_{\mathrm{FH}} \sim 5 \mu \mathrm{m}$. Neither is characteristic of any length scale experimentally observed in the

67 specimens, whether structural or microstructural. For this reason, and because of the lack

68 of any explicit connection between the theories and dislocation dynamics, Evans and

69 Hutchinson noted that strain-gradient theories have not been comprehensively embraced

$70 \quad[23]$.

71 Here, the FH characteristic length is derived from critical thickness theory. This

72 reveals a previously unsuspected link between the two theories. In particular, it provides

73 the explicit connection between the FH theory and the physics of dislocation dynamics

74 that was previously lacking. It thereby validates the use of the FH theory for prediction in

75 engineering applications (with due attention to the approximations revealed in it). 
77 and Hutchinson [23] as a starting-point. They define an effective stress $\sigma$ which is a

78 function of the yield stress and the plastic strain, $\sigma=\sigma_{Y} f\left(\varepsilon_{P}\right)$. For the FH theory, they

79 state as a premise that the plastic work per unit volume may be written as

$$
U_{P}=\sigma_{Y} \int_{0}^{E_{P}} f\left(\varepsilon_{P}\right) d \varepsilon_{P}
$$

81 The upper integral limit $E_{P}$ brings in the effect of the strain gradient $\varepsilon_{P}^{\prime}$ by the definition

$$
E_{P}=\varepsilon_{P}+\ell_{F H} \varepsilon_{P}^{\prime}
$$

83 This is a specific form of the generalized effective plastic strain $E_{p}$ [19]. Consider an

84 object of size $h$, average plastic strain $\bar{\varepsilon}_{P}$ and average plastic strain gradient $\bar{\varepsilon}_{P}^{\prime}=c \bar{\varepsilon}_{P} / h$

85 with $c \sim 1$, and with perfect plasticity, $f\left(\varepsilon_{P}\right)=1$. From equation (1), the average flow

86 stress is

$$
\bar{\sigma}=\sigma_{Y}\left(1+\frac{c \ell_{F H}}{h}\right)
$$

88 This is equation (11) of Ref.23. Note that the strengthening is independent of $\varepsilon P$. The

89 strain gradient increases the yield strength but not the rate of strain-hardening. Using $\ell_{F H}$

$90=5 \mu \mathrm{m}$ and adding a work-hardening term, Evans and Hutchinson [23] obtain excellent

91 fits to the data of Ehrler et al. [8] for nickel foils.

92 We apply equation (3) to simple and very well understood examples of the size

93 effect. These are the plastic relaxation of non-lattice-matched epitaxial strained-layer 
94 structures grown above their critical thicknesses. Growth is in the $z$ direction to a

95 thickness $h$ above the substrate at $z=0$. At typical growth temperatures of $600^{\circ} \mathrm{C}$ for

96 GaAs-based structures (more than half the melting-point) the intrinsic yield strength is

97 very low. The ability to support elastic strains of 0.01 and more at thicknesses of tens of

$98 \mathrm{~nm}$ comes from the size effect. In good-quality growth, there is little or no evidence of

99 work-hardening and the material may be taken to be perfectly plastic. Matthews critical

100 thickness theory [20-22] gives the critical thickness $h_{C}$ at which misfit dislocations

101 (GNDs) may form at $z=0$ to relieve the elastic strain in a simple layer with misfit strain

$102 \varepsilon_{0}$. The result, for our purposes here, is best expressed by the geometrical version of

103 Matthew's theory $[25,26]$, as $h_{C} \sim b / \varepsilon_{0}$ where $b$ is the relevant (in-plane) component of

104 the Burgers vector of the misfit dislocations (the GNDs). This version agrees well with

105 experiment. Moreover, it omits unnecessary detail which is specific to single-crystal

106 cubic semiconductors and also it omits the ill-defined parameters, the inner and outer cut-

107 off radii, that appear in the calculation of the dislocation self-energy. The elastic strain $\varepsilon_{E}$

$108=\varepsilon_{0}$ for $h<h_{C}$ and the plastic relaxation at greater thicknesses gives $\varepsilon_{E} \sim b / h$ for $h>h_{C}$.

109 The condition for plastic relaxation may be written in terms of the strain-thickness

110 product as $\varepsilon_{E} h \sim b$. The theory is readily generalised to more complicated structures

111 (graded layers with $\varepsilon_{0}=g z$, multilayers and superlattices) by considering the strain-

112 thickness integral of $\varepsilon_{E}(z) d z$ over the thickness and introducing plastic relaxation during

113 growth as necessary to limit the integral to the value $b$ [27]. Any intrinsic or bulk strength

114 simply adds to this size-effect strength. In all cases the size effect is due to the energy

115 required to create the length of GND needed to accommodate the misfit. 
116 For significant plastic deformation (stress relaxation) when the initial dislocation

117 density is low, dislocation multiplication must take place - sources must operate.

118 Beanland showed that this requires a much greater thickness, $h_{R} \sim 5 h_{C}$ for simple layers

$119[28,29]$. In this case, the energy required to create the GNDs is small compared with the

120 energy dissipated in source operation. Then the strain-thickness product or integral during

121 plastic deformation is $\sim 5 b$ for $h>h_{R}$. Experimentally, these predictions of the theory

122 have been confirmed extensively in simple layers, graded layers and in more complicated

123 structures [30-32]. The theory also predicts the spatial distribution of GNDs and of $\varepsilon_{P}$

124 [32], confirmed by discrete dislocation dynamics simulation [33].

125 We calculate the average plastic strain, the average plastic strain gradient, the

126 average stress, and the constant $c$ for three standard epitaxial structures (Table I). For the

127 simple constant-composition strained layer with misfit strain $\varepsilon_{0}$ grown above its

128 relaxation critical thickness the plastic strain $\varepsilon_{P}(z)$ throughout the thickness of the layer is

129 constant and so this is also the average, $\bar{\varepsilon}_{P}=\varepsilon_{P}$. The average stress is $\bar{\sigma}=M \varepsilon_{E}$ where $M$

130 is the relevant elastic modulus. The plastic strain gradient is ideally infinite at the

131 substrate - layer interface and zero elsewhere, but the average comes just from the

132 change of plastic strain, from 0 at the substrate at $z=0$ to $\varepsilon_{P}$ at the top at $z=h$. The

133 constant $c=1$ in this case by definition. Then the average stress (Table I), with a bulk

134 yield stress $\sigma_{Y}$ added, may be set equal to the average stress predicted by the FH theory in 135 equation (3) giving, 


$$
\bar{\sigma}=\sigma_{Y}\left(1+\frac{c \ell_{F H}}{h}\right)=M \varepsilon_{E}=\sigma_{Y}+\frac{5 M b}{h}
$$

$$
\ell_{F H}=\frac{5 M b}{\sigma_{Y}}=\frac{5 b}{\varepsilon_{Y}}
$$

137 where $\varepsilon_{Y}$ is the yield strain.

138 In linearly-graded layers, with the misfit increasing as $g z$, the strain-thickness

139 integral without plastic relaxation is $1 / 2 g h^{2}$, and the critical thickness $h_{R}$ is given by setting

140 this equal to $5 b$. When growth continues above $h_{R}$, the lower material relaxes completely.

141 A top layer of thickness $h_{R}$ has a uniform $\varepsilon_{P}$ and stress increasing linearly with the slope

$142 M g$. We consider first a thin structure with growth to a thickness $h=h_{R}+\delta(\delta$ small $)$

143 giving constant plastic strain throughout the grade, except for the thin layer of thickness

$144 \delta h$ at the bottom (Table I) which we ignore. Again $c=1$. The stress increases linearly so

145 the average stress is half the surface stress (Table I). Again adding a bulk yield stress $\sigma_{Y}$

146 and equating the average stress with the average stress of equation (3) we have

$$
\begin{aligned}
& \bar{\sigma}=\sigma_{Y}\left(1+\frac{c \ell_{F H}}{h_{R}}\right)=\sigma_{Y}+1 / 2 M g h_{R} \\
& \ell_{F H}=\frac{1 / 2 M g h_{R}^{2}}{\sigma_{Y}}=\frac{5 b}{\varepsilon_{Y}}
\end{aligned}
$$

148 Graded-layer growth to a much greater thickness $h \gg h_{R}$ gives complete plastic

149 relaxation to $\varepsilon_{E}=0, \varepsilon_{P}=g z$ throughout the layer except for a thin region at the top of

150 thickness $h_{R}$ where $\varepsilon_{P}$ is constant and the elastic strain $\varepsilon_{E}$ rises from 0 to $g h_{R}[27,32]$.

151 Neglecting the thin region at the top, the average plastic strain is $1 / 2 g h$, while the average

152 plastic strain gradient is just $g$, so that here $c=2$. The stress is zero except in the thin 
153 region at the top where it rises from zero to $M g h_{R}$, so the stress-thickness integral is

154 constant at $1 \frac{1}{2} M g h_{R}$ and the average stress is obtaining by multiplying by $h_{R} / h$. Again

155 adding a bulk strength $\sigma_{Y}$ and equating the average stress with the average stress of

156 equation (3) we have,

$$
\begin{aligned}
& \bar{\sigma}=\sigma_{Y}\left(1+\frac{c \ell_{F H}}{h}\right)=\sigma_{Y}+\frac{1 / 2 M g h_{C}^{2}}{h} \\
& \ell_{F H}=\frac{1 / 2 M g h_{C}^{2}}{c \sigma_{Y}}=\frac{5 b}{2 \varepsilon_{Y}}
\end{aligned}
$$

All three examples, equations (4-6), give similar results, varying only because of

159 the factor $c$, so we conclude that

$$
\ell_{F H}=\frac{5 b}{c \varepsilon_{Y}}
$$

161 The problem of a linearly-graded layer maps perfectly onto half of the problem of a beam

162 in bending, from the neutral plane to either free surface [33]. Taking typical numerical

163 values for pure nickel and other soft metals, $M \sim 100 \mathrm{GPa}, b \sim 0.25 \mathrm{~nm}$ and yield

164 strengths about $20 \mathrm{MPa}$, gives $\ell_{F H}=3.125 \mu \mathrm{m}$ from equation (6). This is in good

165 agreement with the results from empirical fits (Fig.1).

166 Evans and Hutchinson [23] give values (but not error bars) of $\ell_{F H}$ obtained by

167 fitting the FH theory to data from different authors for indentation of iridium, silver,

168 copper and a superalloy, and to data for bending nickel foils. They note the inverse

169 correlation between the values of $\ell_{F H}$ and the yield strain $\varepsilon_{Y}$ of the material (figure 1), as

170 in equations (4-7). Their tentative interpretation is that $\ell_{F H}$.represents the distance 
171 moved by dislocations between e.g. cell walls or precipitates, which will be reduced as

$172 \sigma_{Y}^{-1}$ in stronger materials. However, this interpretation overlooks the physical origin of

173 the size effect. Moreover, equation (7) predicts the absolute magnitudes of $\ell_{F H}$ very well

174 (figure 1).

175 The presence of $c$, the ratio of the peak value of $\varepsilon_{P}$ to its average value, in the 176 denominator of equation (7) is interesting. Gradient theory fits DDD simulation results

177 better if the characteristic length is allowed to be a variable and to decrease with strain

178 [24]. The graded layers, equations $(5,6)$ show that $c$ varies from 1 at low strain to 2 at

179 high strain, with a concomitant reduction of a factor of 2 in the characteristic length of 180 equation (7).

181 The phenomenological FH and similar strain-gradient theories express the

182 outcomes of the size effect accurately, but using a fitting parameter, the characteristic

183 length, which is not a true characteristic of the material. Evans and Hutchinson [23]

184 attribute equation (3) to the summation of the energy dissipation caused by the movement

185 of statistically-stored dislocations (SSDs) and that due to the movement of GNDs, the

186 second term.

187 Our interpretation of equation (3) is different. From figure 1 and equation (7), the

188 characteristic length is the Matthews critical thickness $h_{C}$ or the relaxation critical

189 thickness $h_{R}$ calculated using the elastic yield strain or flow stress of the material.

190 Equivalently, it is the thickness $h$ at which the size effect doubles the strength of the

191 material. Note that the $\sigma_{Y}$ in the denominator of equation (7) permits rewriting equation

192 (3) as 


$$
\bar{\sigma}=\sigma_{Y}+\frac{c \sigma_{Y} \ell_{F H}}{h}=\sigma_{Y}+\frac{5 M b}{h}
$$

194 so that the inverse dependence of $\ell_{F H}$ on $\sigma_{Y}$ is cancelled by the prefactor $\sigma_{Y}$. This is a

195 very clear indication that the size effect is independent of the phenomena determining the

196 yield strength, such as dislocation and defect densities. The first term does indeed

197 represent whatever dissipative mechanism is responsible for the strength of bulk material

198 without a size effect, such as the movements of SSDs. The second term, however, in the

199 case that source operation is not required $\left(\varepsilon_{E} \sim b / h\right)$, represents the energy stored (not

200 dissipated) by the creation of GND length - the Matthews model [20-22]. In the case that

201 source operation is required $\left(\varepsilon_{E} \sim 5 b / h\right)$, and this is generally the case for significant

202 plastic deformation, the second term represents mostly the energy dissipated by source

203 operation under the $\sim 5 \times$ greater stress required to operate sources within a restricted size

204 compared with the stress required merely to create extra GND length [29, 31]. In this

205 interpretation, it is clear that neither the presence of GNDs nor the presence of a plastic

206 strain gradient are directly responsible for the increased strength when they are present.

207 The increased strength arises from the energy required to create the GNDs or to operate 208 sources.

209 In this context, it is interesting to observe that the Matthews theory $\left(\varepsilon_{E} \sim b / h\right)$ for

210 simple strained layers requires the presence of a substrate, for otherwise misfit

211 dislocations have nowhere to exist. But given the need for dislocation multiplication, the

212 need to operate sources, the relationship $\varepsilon_{E} \sim 5 b / h$ is independent of the presence or

213 absence of a substrate, since two free surfaces with a separation $h$ constrain the curvatures 
214 of dislocations in a source (to more than $\sim h^{-1}$ ) in much the same way as one free surface

215 and a strained-layer - substrate interface or neutral plane does, or indeed the two

216 interfaces of a capped layer. Consequently, equation (7) applies as well to a stand-alone

217 thin foil, wire or micropillar under uniaxial tension or compression as it does to an

218 epitaxial layer on a substrate, or to a foil under bending or a wire under torsion, as long as

219 due attention is paid to the appropriate value of $h$ in each case.

220 In the applications of equations (1-3) the primary unknown is the plastic strain

221 distribution. It can be obtained within the strain-gradient theory by analytic means for

222 very simple cases such as the beam in bending [23], or by numerical methods [19].

223 However, these methods rely upon the approximation that the stress-strain relationship

224 implied by equations (1-3) is local. This is an approximation that is severely in error for

225 the simple strained layer, since only the material at the substrate - layer interface

226 experiences a plastic strain gradient, yet the full thickness of the layer is capable of

227 sustaining the stress $M \varepsilon_{E} \gg \sigma_{Y}$. Source operation and significant plastic deformation do

228 not depend upon conditions at a point, but upon conditions over an extended region

229 (source size) around the point, as recognised in nonlocal plasticity theories. Nevertheless,

230 the approximation can be good - this is best seen in the beam-bending or graded layer

231 problems. That is why, as observed by Liu et al. [10], the experimental data cannot test

232 between critical thickness theory and strain-gradient theory, for both will fit well.

233 It is worth commenting on the possible application of this analysis to other

234 gradient theories. Whenever the gradient term is multiplied by the yield or flow stress, as

235 in equation (3), and then the characteristic length turns out to vary as the inverse of the

236 yield or flow stress (or plastic strain), the separation we have done in equation (8) is 
237 possible. This gives a gradient coefficient unrelated to yield or flow stress and then

238 interpretations in terms of dislocation or defect spacing become inappropriate. From the

239 review by Zhang and K. Aifantis [34], this seems to be the case for most gradient theories

240 including those based on, or equivalent to, the Aifantis theories [24, 35].

241 In conclusion, it is demonstrated that the characteristic length in the FH strain-

242 gradient theory can be obtained from known material and structural parameters,

$243 \ell_{F H}=5 b / c \varepsilon_{Y}, c \sim 1$. The derivation shows that this SGP corresponds physically to

244 critical thickness theory. It explains why SGP theories are capable of fitting experimental

245 data. It validates the use of this theory to obtain approximate constitutive laws for use in

246 finite-element calculations. It offers the prospect of understanding in general, on a secure

247 physical basis, why strong metals are strong, and how to include size effects in rigorous

248 engineering modelling and simulation.

249 Acknowledgements: Undergraduate students David Lewis and Viktor Jevdomikov

250 assisted with strain-gradient theory. Discussions with the late Tony (A.G.) Evans, and

251 with Dabiao Liu, Andy J. Bushby and Alan J. Drew are gratefully acknowledged.

252

253 References

254 1. E.O. Hall, Proc. Phys. Soc. B64, (1951) p.747.

255 2. S.V. Raj and G.M. Pharr, Mater. Sci. Eng. 81 (1986) p.217.

256 3. W.D. Nix and H.J. Gao, J. Mech. Phys. Sol. 46 (1988) p.411.

257 4. N.A. Fleck, G.M. Muller, M.F. Ashby and J.W. Hutchinson, Acta Met. Mat. 42

258 (1994) p.475.

259 5. Q. Ma and D.R. Clarke, J. Mater. Res. 10 (1995) p.853.

260 6. J.S. Stölken and A.G. Evans, Acta Mat. 46, (1998) p.5109.

261 7. M.D. Uchic, D.M. Dimiduk, J.N. Florando and W.D. Nix, Science 305 (2004) p.986. 
8. B. Ehrler, X.D. Hou, T.T. Zhu, K.M.Y. P'ng, C.J. Walker, A.J. Bushby and D.J. Dunstan, Phil. Mag. 88 (2008) p.3043.

9. D.J. Dunstan, B. Ehrler, R. Bossis, S. Joly, K.M.Y. P'ng and A.J. Bushby, Phys. Rev. Lett. 103 (2009) p.155501.

10. D. Liu, Y. He, D.J. Dunstan, B. Zhang, Z. Gan, P. Hu and H. Ding, Int. J. Plasticity 41 (2013) p.30.

11. J.-K. Heyer, S. Brinckmann, J. Pfetzing-Micklich and G. Eggeler, Acta Mat. 62 (2014) p.225.

12. D.J. Dunstan and A.J. Bushby, Int. J. Plasticity 53 (2014) p.56.

13. M. W. Kapp, C. Kirchlechner, R. Pippan and G. Dehm , J. Mater. Res. 30 (2015) p.791.

14. D.J. Dunstan and A.J. Bushby, Int. J. Plasticity 40 (2013) p.152.

15. M.F. Ashby, Phil. Mag. 21 (1970) p.399.

16. E.C. Aifantis, J. Eng. Mater. Tech. - Trans. ASME 106 (1984), p.326.

17. N.A. Fleck and J.W. Hutchinson, J. Mech. Phys. Solids, 41 (1993) p.1825.

18. M.I. Idiart, V.S. Deshpande, N.A. Fleck and J.R. Willis, Int. J. Eng. Sci. 47 (2009) p. 1251.

19. N.A. Fleck, J.W. Hutchinson and J.R. Willis, J. Appl. Mech. 82 (2015) p.071002.

20. J.W. Matthews, S. Mader and T.B. Light, J. Appl. Phys. 41 (1970) p.3800.

21. E.A. Fitzgerald, Mater. Sci. Rep. 7 (1991) p.91.

22. D.J. Dunstan, J. Mater. Sci.: Mater. Electronics 8 (1997) p.337.

23. A.G. Evans and J.W. Hutchinson, Acta Mat. 57 (2009) p.1675.

24. X. Zhang and K.E. Aifantis, Mater. Sci. Eng. A631 (2015) p.27.

25. L. Bragg, Nature 149 (1942) p.511.

26. D.J. Dunstan, S. Young and R.H. Dixon, J. Appl. Phys. 70 (1991) p.3038.

27. D.J. Dunstan, Phil. Mag. A73 (1996) p.1323.

28. R. Beanland, J. Appl. Phys. 72 (1992) p.4031.

29. R. Beanland, J. Appl. Phys. 77 (1995) p.6217.

30. D.J. Dunstan, P. Kidd, L.K. Howard and R.H. Dixon, Appl. Phys. Lett. 59 (1991) p.3390.

31. D.J. Dunstan, P. Kidd, R. Beanland, A. Sacedón, E. Calleja, L. González, Y. González and F.J. Pacheco, Mater. Sci. Technol. 12 (1996) p.181.

32. J. Tersoff, Appl. Phys. Lett. 62 (1993) p.693.

33. C. Motz and D.J. Dunstan, Acta Mat. 60 (2012) p.1603.

34. X. Zhang and K. Aifantis, Rev. Adv. Mater. Sci. 41 (2015) p.72. 
297 35. E.C. Aifantis, Mechanics of Materials, 35 (2003) p. 259.

298 
299 Table I. Parameters in the critical thickness calculations for strained layers with $\sigma_{Y}=0$. Symbols are defined in the text.

\begin{tabular}{|l|c|c|c|c|c|c|c|c|c|}
\hline Structure & $\varepsilon(z)$ & $H_{R}$ & $h$ & $\varepsilon_{E}(z)$ & $\bar{\sigma}$ & $\varepsilon_{P}(z)$ & $\bar{\varepsilon}_{P}$ & $\bar{\varepsilon}_{P}^{\prime}$ & $c$ \\
\hline Simple layer & $\varepsilon_{0}$ & $5 b / \varepsilon_{0}$ & $>h_{R}$ & $5 b / h$ & $5 M b / h$ & $\varepsilon_{0}-\varepsilon_{E}$ & $\varepsilon_{P}$ & $\varepsilon_{P} / h$ & 1 \\
\hline Thin grade & $g z$ & $\sqrt{10 b / g}$ & $h_{R}+\delta$ & $\begin{array}{l}z<\delta: 0 \\
\text { else: } g(z-\delta)\end{array}$ & $\sim 1 / 2 M g h_{R}$ & $\begin{array}{l}z<\delta: g z \\
\text { else: } g \delta\end{array}$ & $\sim g \delta$ & $\sim g \delta / h$ & $\sim 1$ \\
\hline Thick grade & $g z$ & $\sqrt{10 b / g}$ & $>h_{R}$ & $\begin{array}{l}\begin{array}{l}z<\left(h-h_{R}\right): 0 \\
\text { else: } g\left(z-h+h_{\mathrm{R}}\right)\end{array} \\
\sim 1 / 2 M g \frac{h_{R}^{2}}{h}\end{array}$ & $\begin{array}{l}z<\left(h-h_{R}\right): g z \\
\text { else: } g\left(h-h_{R}\right)\end{array}$ & $\sim 1 / 2 g h$ & $\sim g$ & $\sim 2$ \\
\hline
\end{tabular}

300 


\section{$301 \quad$ Figure Caption}

302 Figure 1. Characteristic lengths $\ell_{F H}$ are plotted against the tensile yield strains $\varepsilon_{Y}$. The

303 length scales were found by fitting the FH theory to indentation data from the literature

304 for $\mathrm{Ir}, \mathrm{Ag}, \mathrm{Cu}$ and superalloy and to foil-bending data for Ni. After figure 13 of reference

305 23. The solid line is the prediction of equation (7), for a typical value of $b=0.25 \mathrm{~nm}$ and 306 with $c=2$.

307 